\title{
Magnetic intensification of spectral lines
}

\author{
M. J. Stift ${ }^{1}$ and F. Leone ${ }^{2}$ \\ ${ }^{1}$ Institut für Astronomie (IfA), Universität Wien, Türkenschanzstrasse 17, 1180 Wien, Austria \\ 2 INAF, Osservatorio Astrofisico di Catania, Via S. Sofia n. 78, 95123 Catania, Italy \\ e-mail: fleone@ct.astro.it
}

Received 26 June 2002 / Accepted 25 October 2002

\begin{abstract}
We present an in-depth investigation of the increase in equivalent width of saturated lines under the influence of strong magnetic fields, aptly called "magnetic intensification". Assuming 20 different Zeeman patterns (with the number of components ranging from 3 to 45 ) for a fictitious $\mathrm{Fe}$ II line at $4500 \AA$ in a $10000 \mathrm{~K}, \log g=4.0 \mathrm{Kurucz}$ atmosphere, we calculate equivalent widths as a function of magnetic field strength, field angle and line strength (abundance). The increase over the zero field equivalent width is found to always fall below the $n / 2$ relation suggested by Babcock (1949), where $n$ is the number of Zeeman components. After a discussion of the behaviour of various Zeeman patterns we turn to the influence of anomalous dispersion on magnetic intensification. Changes in line blanketing due to magnetic desaturation appear to be of negligible size. Finally we show that magnetic intensification leads to apparent enhanced abundances and to virtual abundance variations in magnetic stars with dipolar surface magnetic field configurations.
\end{abstract}

Key words. stars: abundances - stars: chemically peculiar - stars: magnetic fields - polarization

\section{Introduction}

In an important paper, Babcock (1949) realised that the Zeeman patterns of many absorption lines in the spectra of chemically peculiar stars on the upper main sequence (CP stars) which are permeated by strong magnetic fields (some $10 \mathrm{mT}$ to $3.5 \mathrm{~T}$ ) must be considerably wider than the thermal Doppler profiles, leading to a marked increase in the total absorption of saturated lines. Such an increase should become particularly important in lines having wide anomalous Zeeman patterns of many components, and Babcock surmised that the apparent over-abundances of several chemical elements and the large intensity variations of some lines of these elements might mainly or even exclusively be due to this aptly called "magnetic intensification".

Shortly afterwards, Warwick (1951) noted the occurrence of non zero net polarisation in spectral lines subject to magnetic intensification but it was Leroy (1962) who first applied these findings to explain the observed polarisation of sunspot radiation and who suggested (Leroy 1989) that broadband linear polarisation (BBLP) might be detectable in magnetic stars. Polarimetric observations of $55 \mathrm{CP}$ stars were collected over the years (Leroy et al. 1993; Leroy 1995) and attempts were made to interpret them in terms of a simple canonical model developed by Landolfi et al. (1993).

Send offprint requests to: M. J. Stift, e-mail: stift@astro.univie.ac.at
The correct modelling of the spectra of the $\mathrm{CP}$ stars no doubt requires a deep understanding of magnetic intensification, but nobody ever has carried out an exhaustive study of, for example, the over-estimation of elemental abundances in the presence of strong, large-scale magnetic fields, nor has the behaviour of polarised blends ever been investigated in sufficient detail. The same is true for the impact of magneto-optical effects on abundance determinations in CP stars - many modelling attempts have neglected the Faraday terms in the polarised radiative transfer equation. Let us also note that the role of magnetic intensification in Doppler Imaging based solely on intensity spectra is not yet completely understood; Stift (1996) found that spurious abundances in virtual patches are pure artefacts of the regularisation function, only very remotely related to magnetic intensification and in this sense unphysical.

Large new atomic databases and the recently developed object-oriented code COSSAM (Stift 2000) which incorporates the best affordable input physics and which can execute on massively parallel multiprocessor machines have made it possible to address all these questions. In the present paper we want to provide new insight into the workings of magnetic intensification, mainly for single lines but also for (heavily) blended spectra. A close look at magneto-optical effects, on how they modify the Stokes IQUV spectrum and affect abundance determinations, is also warranted in this context. As to broadband linear and circular polarisation which accompany magnetic intensification, they will be the subject of a separate paper. For a 
Table 1. Zeeman patterns for the 20 transitions used in the present investigation together with the spectroscopic terms from which the transitions originate, their respective Lande factors, $g_{\text {eff }}$ the center of gravity of the $\sigma_{+}$components in Lorentz units, and the number of the $\sigma_{+}$and the $\pi$ components.

\begin{tabular}{rccccrr}
\hline \hline Nos. & transition & $g_{\text {low }}$ & $g_{\text {up }}$ & $g_{\text {eff }}$ & $n(\sigma)$ & $n(\pi)$ \\
\hline 0 & ${ }^{1} \mathrm{P}_{1}-{ }^{1} \mathrm{P}_{1}$ & 1.00 & 1.00 & 1.00 & 1 & 1 \\
1 & ${ }^{2} \mathrm{P}_{3 / 2}-{ }^{2} \mathrm{~S}_{1 / 2}$ & 1.33 & 2.00 & 1.67 & 2 & 2 \\
2 & ${ }^{2} \mathrm{G}_{9 / 2}-{ }^{2} \mathrm{~F}_{7 / 2}$ & 1.11 & 1.14 & 1.06 & 8 & 8 \\
3 & ${ }^{2} \mathrm{D}_{3 / 2}-{ }^{2} \mathrm{P}_{1 / 2}$ & 0.80 & 0.67 & 0.83 & 2 & 2 \\
4 & ${ }^{2} \mathrm{G}_{7 / 2}-{ }^{2} \mathrm{~F}_{5 / 2}$ & 0.89 & 0.86 & 0.93 & 6 & 6 \\
5 & ${ }^{8} \mathrm{~F}_{7 / 2}-{ }^{8} \mathrm{D}_{5 / 2}$ & 1.62 & 2.06 & 1.07 & 6 & 6 \\
6 & ${ }^{6} \mathrm{~F}_{5 / 2}-{ }^{6} \mathrm{D}_{3 / 2}$ & 1.31 & 1.87 & 0.90 & 4 & 4 \\
7 & ${ }^{2} \mathrm{G}_{7 / 2}-{ }^{2} \mathrm{~F}_{7 / 2}$ & 0.89 & 1.14 & 1.02 & 7 & 8 \\
8 & ${ }^{2} \mathrm{D}_{3 / 2}-{ }^{2} \mathrm{P}_{3 / 2}$ & 0.80 & 1.33 & 1.07 & 3 & 4 \\
9 & ${ }^{5} \mathrm{D}_{1}-{ }^{5} \mathrm{~F}_{1}$ & 1.50 & 0.00 & 0.75 & 2 & 2 \\
10 & ${ }^{3} \mathrm{D}_{2}-{ }^{3} \mathrm{P}_{1}$ & 1.17 & 1.50 & 1.00 & 3 & 3 \\
11 & ${ }^{3} \mathrm{D}_{1}-{ }^{3} \mathrm{P}_{1}$ & 0.50 & 1.50 & 1.00 & 2 & 2 \\
12 & ${ }^{3} \mathrm{D}_{1}-{ }^{3} \mathrm{D}_{1}$ & 0.50 & 0.50 & 0.50 & 1 & 1 \\
13 & ${ }^{5} \mathrm{D}_{2}-{ }^{5} \mathrm{~F}_{2}$ & 1.50 & 1.00 & 1.25 & 4 & 4 \\
14 & ${ }^{7} \mathrm{D}_{2}-{ }^{7} \mathrm{~F}_{3}$ & 2.00 & 1.50 & 1.00 & 5 & 5 \\
15 & ${ }^{6} \mathrm{D}_{5 / 2}-{ }^{6} \mathrm{~F}_{5 / 2}$ & 1.66 & 1.31 & 1.49 & 5 & 6 \\
16 & ${ }^{7} \mathrm{~S}_{3}-{ }^{7} \mathrm{P}_{3}$ & 2.00 & 1.92 & 1.96 & 6 & 6 \\
17 & ${ }^{9} \mathrm{H}_{6}-{ }^{9} \mathrm{G}_{5}$ & 1.38 & 1.50 & 1.08 & 11 & 11 \\
18 & ${ }^{9} \mathrm{D}_{6}-{ }^{9} \mathrm{~F}_{5}$ & 1.67 & 1.63 & 1.75 & 11 & 11 \\
19 & ${ }^{9} \mathrm{I}_{7}-{ }^{9} \mathrm{H}_{8}$ & 1.30 & 1.43 & 1.87 & 15 & 15 \\
\hline
\end{tabular}

gentle introduction to polarised radiative transfer the reader is referred to Rees (1987).

\section{COSSAM: Codice per la Sintesi Spettrale nelle Atmosfere Magnetiche}

The exploration of magnetic intensification and broadband polarisation in realistic, heavily blended stellar spectra must be based on extensive atomic transition data, accurate and stable numerical algorithms, and massive computational resources. No approximate treatment of anomalous Zeeman patterns in heavily blended lines can replace full opacity sampling over the $\sigma_{-}$, the $\sigma_{+}$, and $\pi$ components separately. And since the radiative transfer cannot be adequately described by the diffusion approximation in the outer layers of the $\mathrm{CP}$ stars, the radiative transfer equation has to be solved with a stable and accurate formal solver, best with the Zeeman-Feautrier method of Auer et al. (1977).

When high polarimetric accuracy is required, the spectrum must be sampled at least every $10 \mathrm{~m} \AA$. This leads to $10^{5}$ frequency points for the modelling of the observations of Leroy (1995) in which filters were used which cover wavelength intervals of $1000 \AA$ and more. Solving the polarised transfer equation $10^{5}$ times (with about 100 points in optical depth) in addition to sampling the opacity of several $10^{5}$ Zeeman components at each frequency point is extremely time-consuming and outside the reach of even the fastest single-processor machines if

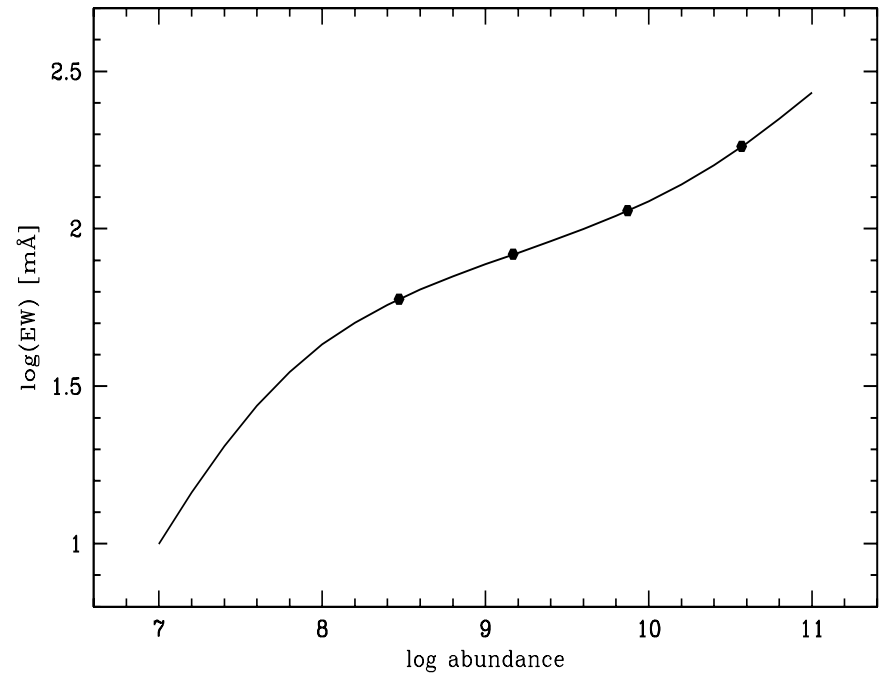

Fig. 1. Curve of growth of the fictitious iron line at $\lambda 4500$ with the 4 abundances adopted in the present investigation and the corresponding equivalent widths indicated. Abundances are relative to a hydrogen abundance $\log \epsilon_{\mathrm{H}}=12.00$.

one considers that this has to be done for each point of a sometimes fairly dense spatial grid covering the visible hemisphere of a magnetic stars. Thus only (massively) parallel computing offers the possibility of a realistic modelling of broadband polarisation in stellar atmospheres permeated by magnetic fields. The same holds for any investigation of how line blanketing is modified by magnetic intensification.

The physical, numerical and computational requirements outlined above could not be met by any existing line synthesis code, so a new code had to be developed. COSSAM is an object-oriented and parallel polarised spectrum synthesis code. For a description see Stift (2000), Wade et al. (2001) and Alecian \& Stift (2002). COSSAM is written entirely in Ada95, the only standardised (ISO/IEC 8652:1995) object-oriented language with concurrent constructs. It takes advantage of Ada's thread-parallel approach which is perfectly suited to the "embarrassingly parallel" nature of the spectral line synthesis problem. There is no need for Message Passing Interfaces (MPI) as in Fortran. A detailed discussion of concurrency and light-weight synchronisation with Ada95 in this astrophysical context has been presented by Stift (1998b). COSSAM is based on truly reusable software components which rely heavily on abstract data types, encapsulation and information hiding, generics, inheritance and programming by extension; more details on Ada95 in (astro)physics can be found in Stift (1998a).

\section{Magnetic intensification as a function of line strength and of Zeeman pattern}

As mentioned right in the beginning, Babcock (1949) surmised that lines with very broad and complicated Zeeman patterns are subject to exceptionally large magnetic intensification: the equivalent width $W$ of a strongly saturated spectral line, fully split into $n$ Zeeman components, would exceed the zero field 


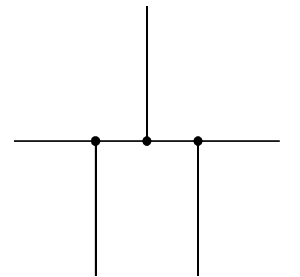

${ }^{1} \mathrm{P}_{1}{ }^{1} \mathrm{P}_{1}(0)$

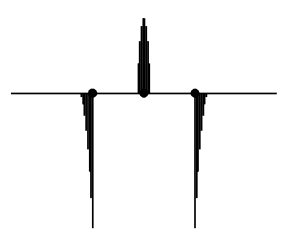

${ }^{2} \mathrm{G}_{9 / 2}{ }^{2} \mathrm{~F}_{7 / 2}$

(2)
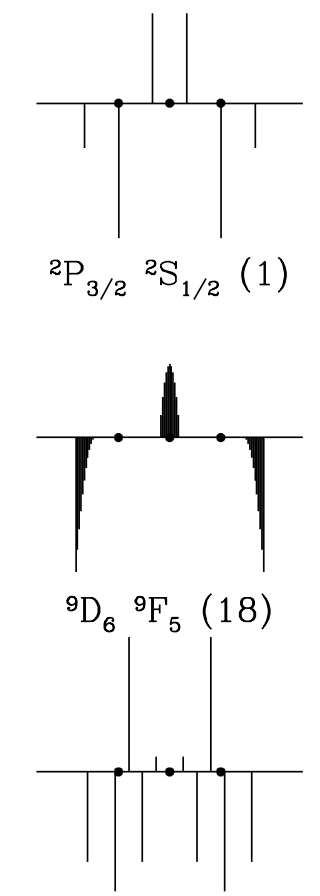

${ }^{7} \mathrm{~S}_{3}{ }^{7} \mathrm{P}_{3}(16)$

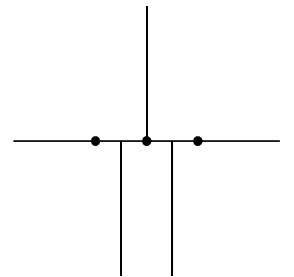

${ }^{3} \mathrm{D}_{1}{ }^{3} \mathrm{D}_{1}(12)$
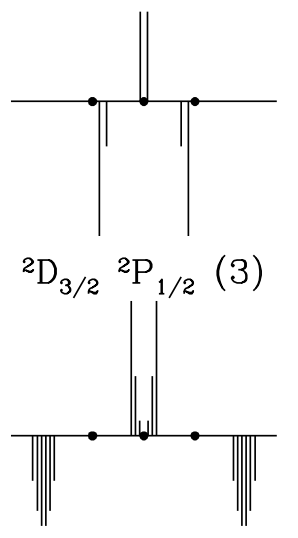

${ }^{2} \mathrm{D}_{3 / 2}{ }^{2} \mathrm{P}_{3 / 2}$

(8)

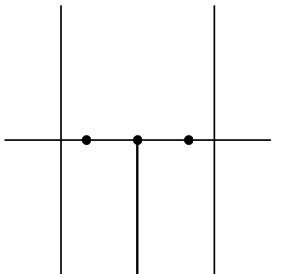

${ }^{5} \mathrm{D}_{1}{ }^{5} \mathrm{~F}_{1}(9)$

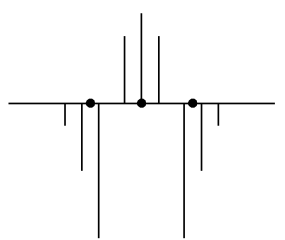

${ }^{3} \mathrm{D}_{2}{ }^{3} \mathrm{P}_{1}(10)$
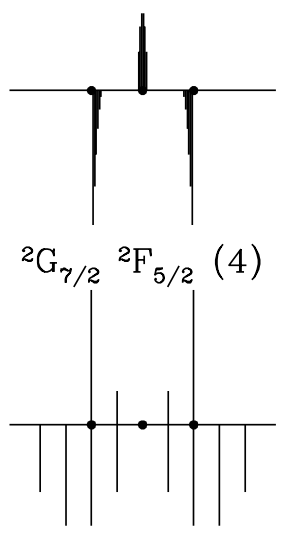

${ }^{5} \mathrm{D}_{2}{ }^{5} \mathrm{~F}_{2}(13)$

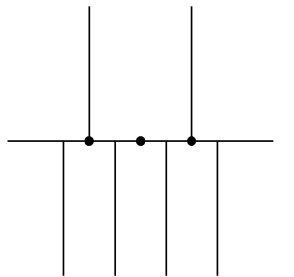

${ }^{3} \mathrm{D}_{1}{ }^{3} \mathrm{P}_{1}(11)$

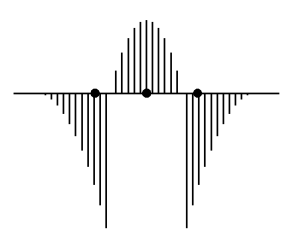

${ }^{9} \mathrm{H}_{6}{ }^{9} \mathrm{G}_{5}$ (17)

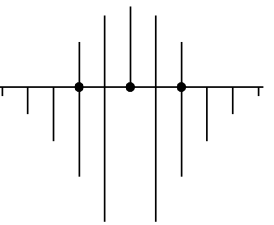

${ }^{7} \mathrm{D}_{2}{ }^{7} \mathrm{~F}_{3}(14)$

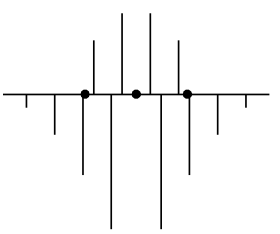

${ }^{6} \mathrm{~F}_{5 / 2}{ }^{6} \mathrm{D}_{3 / 2}(6)$
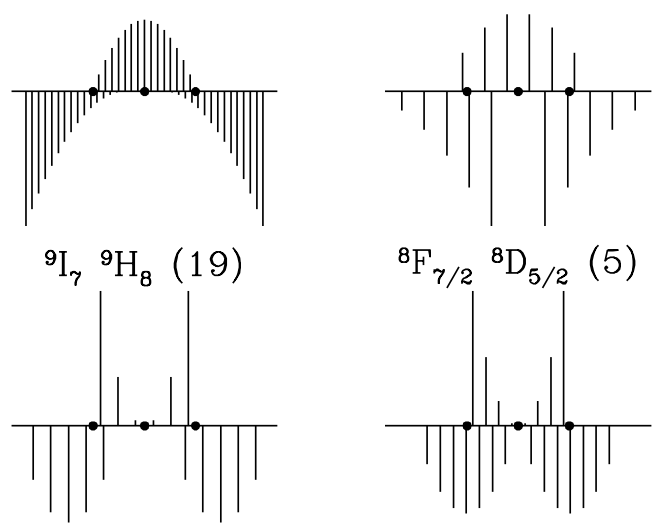

${ }^{6} \mathrm{D}_{5 / 2}{ }^{6} \mathrm{~F}_{5 / 2}$

(15)

${ }^{2} \mathrm{G}_{7 / 2}{ }^{2} \mathrm{~F}_{7 / 2}$

Fig. 2. The 20 Zeeman patterns used in the present investigation. The $\pi$ - and the $\sigma$-components are displayed the usual way, above and below the horizontal line respectively. Dots represent the positions of the components of a simple Zeeman triplet.

equivalent width $W_{0}$ by a factor of up to $n / 2$. Accordingly, the moderate magnetic intensification $W / W_{0}$ in simple triplets would grow to absolutely spectacular proportions in lines with e.g. 50 Zeeman components. To our knowledge, this prediction has never been verified quantitatively, nor has there been a quantitative assessment of the influence of magneto-optical on the predicted factor of $n / 2$.

For the first part of our systematic investigation, we have taken a fictitious iron line at $4500 \AA$, setting the abundance to 4 different values such that the respective zero field equivalent widths are $60,83,114$ and $182 \mathrm{~m} \AA$ in a $10000 \mathrm{~K}, \log g=4.0$ Kurucz (1993) model atmosphere. Figure 1 shows to what part of the curve of growth these 4 equivalent width values belong. Assuming 20 different Zeeman patterns with the number of components ranging from 3 to 45 , we have calculated a dense grid ( 66 field strengths between 0 and $13 \mathrm{~T}, 46$ field angles between $0^{\circ}$ and $90^{\circ}$ ) of integrated Stokes profiles and of equivalent widths in an $8 \AA$ interval centred around $4500 \AA$. Figure 2 displays the Zeeman patterns in question and Table 1 lists some of their basic characteristics.

\subsection{Maximum magnetic intensification}

Our guess is that Babcock's (1949) prediction will hold only if it holds at all - for highly saturated lines located at the lower end of the square root part of the curve of growth, but that lines with a large number of Zeeman components will probably not even under these circumstances qualify for an intensification by a factor of $n / 2$. The argument is at follows: any line that splits into say 40 components, each of which remains still saturated in the Doppler core, would exhibit strong wings. Conversely, the 40 components of a line without strong wings would never remain highly saturated. As a natural consequence, the magnetic intensification of a line of given equivalent width should flatten out at large values of $n$, the number of Zeeman components. Needless to say that the respective intensifications encountered in moderately saturated lines of 60 or $83 \mathrm{~m} \AA$ equivalent width will not match those of the strong lines with 114 or $182 \mathrm{~m} \AA$.

We have limited our investigation to field strengths up to $13 \mathrm{~T}$ mainly for 2 reasons. First, observed mean field moduli in CP stars range from a few tenths of Teslas to at most $3.5 \mathrm{~T}$ 

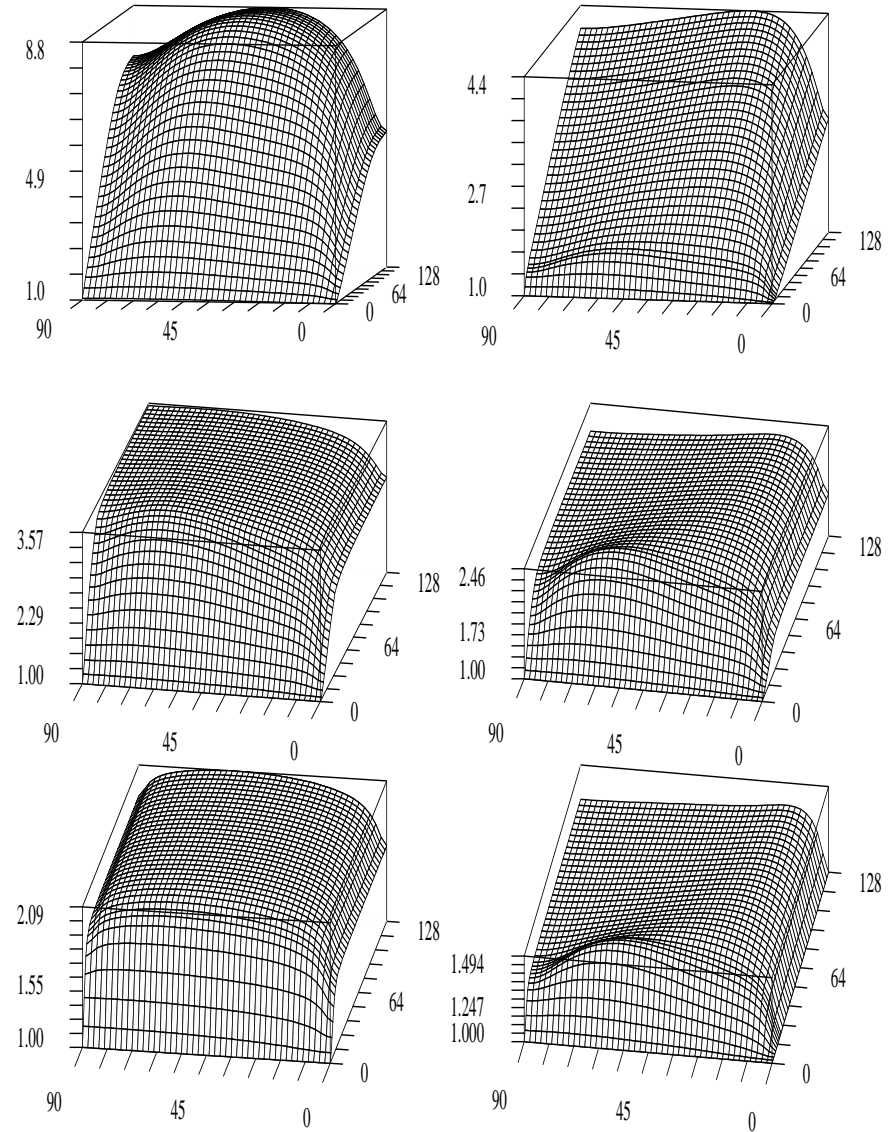

Fig. 3. Magnetic intensification as a function of magnetic field strength and field angle of the fictitious iron line at $\lambda 4500$ with $182 \mathrm{~m} \AA$ equivalent width, assuming various Zeeman patterns. From left to right and from top to bottom we show patterns Nos. 17, 16, 13, 1, 9, and 0. Angles are given in degrees, field strengths are in non-standard kGauss $(100 \mathrm{mT})$ in order to improve the display.

in Babcock's star; so even taking into account decentred dipole geometries it is unlikely that anywhere on the surface of a $\mathrm{CP}$ star there will be fields in excess of $13 \mathrm{~T}$. Secondly, at such high field strengths transition to the Paschen-Back regime will certainly occur in many lines, invalidating our LS coupling approximation.

The dependence of equivalent width from Zeeman pattern, field strength and field angle varies in interesting ways, although it does not present major surprises. Patterns Nos. 2, 4, 16 , and 18 are found to reach maximum equivalent width well beyond $13 \mathrm{~T}$ for all 4 abundances in question. Figure 3 illustrates the characteristic behaviour of the fictitious iron line with $182 \mathrm{~m} \AA$ zero-field equivalent width for 6 different patterns, viz. patterns Nos. $0,1,9,13,16$, and 17. Magneto-optical effects are conspicuous for patterns Nos. 0 and 1, just visible for patterns Nos. 13 and 16, hardly discernible as far as patterns Nos. 9 and 17 are concerned. Whereas a classical triplet experiences no magnetic intensification at all in a longitudinal field, this is no longer true in the case of more complex patterns. Maximum intensification occurs at different angles, ranging from $22^{\circ}$ to $90^{\circ}$ and at field strengths between 0.8 and $12.8 \mathrm{~T}$. A listing of the characteristics of magnetic intensification can be found in Table 2.

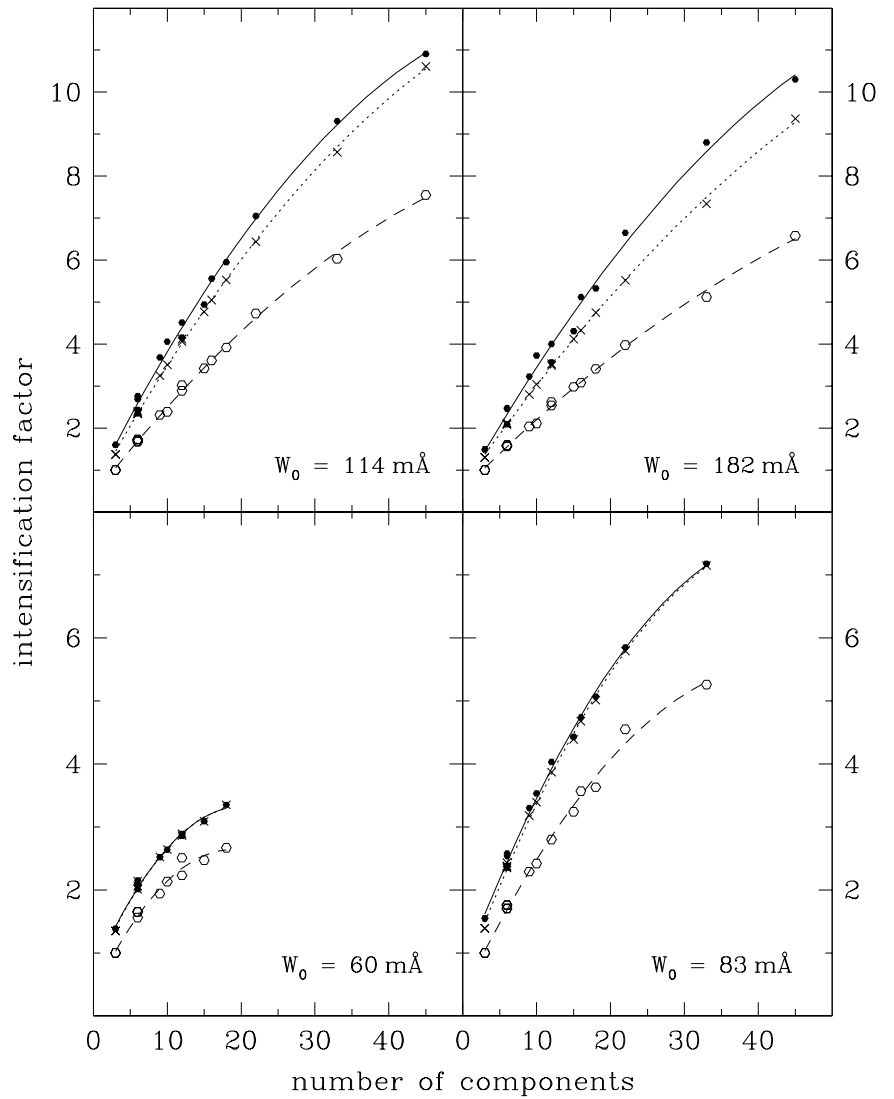

Fig. 4. Maximum magnetic intensification $W_{\max } / W_{0}$ as a function of the number of Zeeman components of the fictitious iron line at $\lambda 4500$ with $60,83,114$, and $182 \mathrm{~m} \AA$ equivalent width (full lines and filled circles). $W n_{\max } / W_{0}$ is similar but without magneto-optical effects taken into account (dotted lines and diagonal crosses). Dashed lines and open circles pertain to $W l_{\max } / W_{0}$, the maximum intensification in a longitudinal field. Note that for the $60 \mathrm{~m} \AA$ line, maximum intensification occurs beyond $13 \mathrm{~T}$ when 20 or more Zeeman components are present, leading to the conspicuous cutoff in the corresponding curve.

With 33 Zeeman components, the equivalent width of pattern No. 17 should increase 16.5 fold over the zero-field value but we find a factor of 8.8 only. Despite a predicted factor of 6 , pattern No. 13 provides a mere 3.6 fold increase. The overall maximum is represented by a 10.9 fold increase for pattern No. 19 with 45 components for a $114 \mathrm{~m} \AA$ zero-field equivalent width; it drops to a value of 10.3 for the $182 \mathrm{~m} \AA$ line, a behaviour shared by all other patterns. This is in most gratifying agreement with our expectations lined out above.

On the other hand, in longitudinal fields one may encounter intensification values of up to 7.5 (again pattern No. 19). For a given zero-field equivalent width, the variation in the ratio between maximum equivalent width $W_{\max }$ and maximum longitudinal equivalent width $W l_{\max }$ lies in a rather narrow band: from $1.13-1.38$ for the weakest line to $1.34-1.76$ for the strongest line.

A simple second order polynomial in $n$, the total number of Zeeman components, fits the intensification of the Zeeman patterns which reach maximum equivalent width below $13 \mathrm{~T}$ and 
we derive for the $60,83,114$, and $182 \mathrm{~m} \AA$ lines respectively (in this order)

$$
\begin{aligned}
& W_{\max } / W_{0}=0.6973+0.2604 n-0.00644 n^{2} \\
& W_{\max } / W_{0}=0.7256+0.3070 n-0.00340 n^{2} \\
& W_{\max } / W_{0}=0.5822+0.3495 n-0.00265 n^{2} \\
& W_{\max } / W_{0}=0.5367+0.3109 n-0.00203 n^{2}
\end{aligned}
$$

where $W_{\max }$ is the maximum equivalent width over all field strengths and angles, and $W_{0}$ the zero field equivalent width.

$$
\begin{aligned}
& W l_{\max } / W_{0}=0.3541+0.2433 n-0.00648 n^{2} \\
& W l_{\max } / W_{0}=0.3291+0.2431 n-0.00280 n^{2} \\
& W l_{\max } / W_{0}=0.3865+0.2258 n-0.00151 n^{2} \\
& W l_{\max } / W_{0}=0.5369+0.1754 n-0.00095 n^{2}
\end{aligned}
$$

with $W l_{\max }$ the maximum over all field strengths for a longitudinal field. Figure 4 displays these results in graphical form.

The maximum slope encountered is about 0.35 , much below the value postulated by Babcock. In addition, the negative quadratic term is quite important, bringing down the slope even further at large values of $n$ the number of components.

\subsection{The importance of magneto-optical effects}

Magneto-optical effects can cyclically convert the Stokes $Q, U$ and $V$ parameters among each other as a result of anomalous dispersion, if there is overlap between the $\pi$ - and the $\sigma$-components. This has of course repercussions on the intensity (Stokes $I$ ) and hence on the equivalent width of a line. In fact, one can easily see that when the saturation in a strong $\pi$-component diminishes (the weak $\sigma$-components becoming stronger at the same time) there must be an increase in total absorption of the line. The same mechanism also works in the other direction, of course at different angles of the magnetic field vector. Our calculations reveal, as expected and shown in Fig. 4, that magneto-optical effects become particularly important in strong lines, whereas in lines of moderate equivalent width maximum intensification $W n_{\max }$ hardly differs from $W_{\max }$. A polynomial fit with $n$ yields

$$
\begin{aligned}
& W n_{\max } / W_{0}=0.6501+0.2682 n-0.00674 n^{2} \\
& W n_{\max } / W_{0}=0.5805+0.3124 n-0.00345 n^{2} \\
& W n_{\max } / W_{0}=0.5289+0.3155 n-0.00206 n^{2} \\
& W n_{\max } / W_{0}=0.6248+0.2519 n-0.00132 n^{2} .
\end{aligned}
$$

These results by no means imply that magneto-optical effects have a negligible influence on the equivalent widths of moderately strong lines. On the contrary, they can lead to a substantial increase in equivalent widths at certain angles and even modest field strengths.

Figure 5 displays, for selected Zeeman patterns, the percental increase in equivalent width due to magneto-optical effects, defined as $R=100(W-W n) / W_{n}$, as a function of magnetic angle and field strength. Already for the $83 \mathrm{~mA}$ line, this increase can attain some $13 \%$, reaching an impressive maximum of $23 \%$ in the case of the $182 \mathrm{~m} \AA$ line. More systematically, we note that the sensitivity to magneto-optical effects
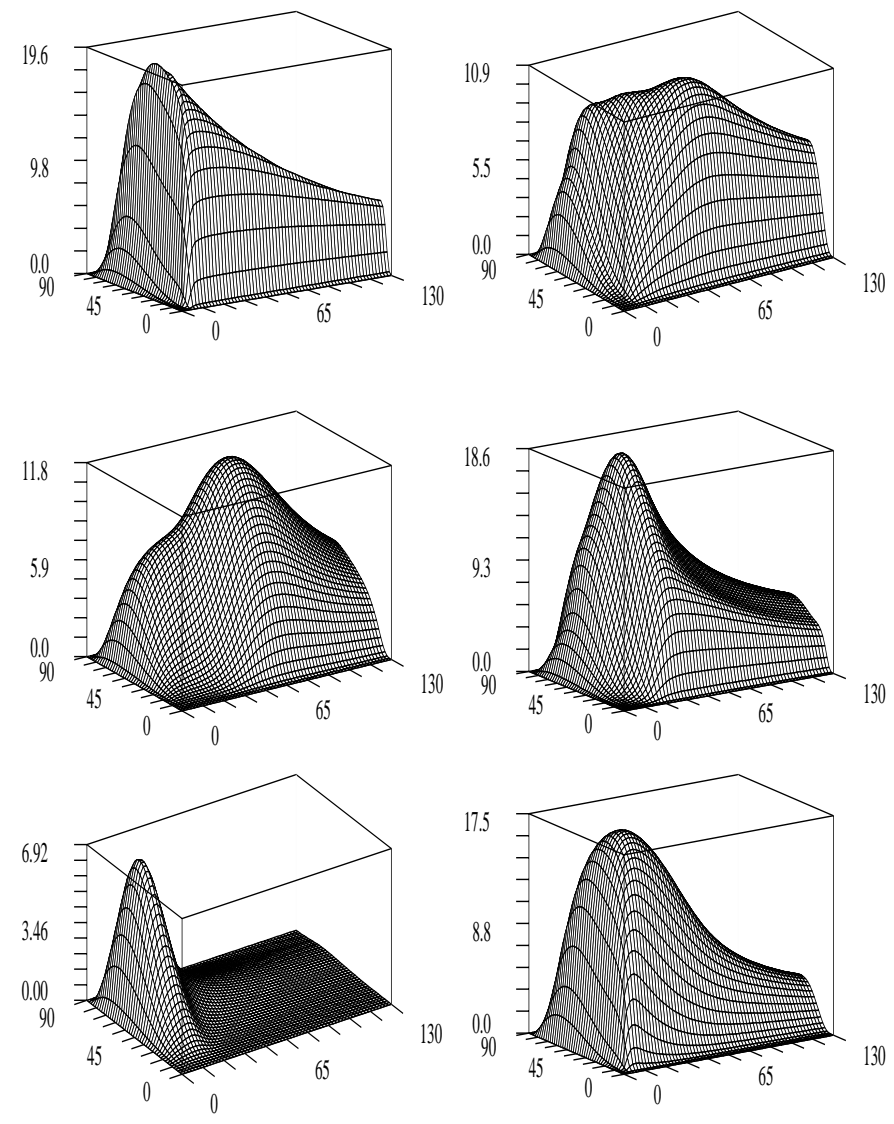

Fig. 5. Percental increase in magnetic intensification $100(W-W n) / W_{n}$ - as a function of magnetic field strength and field angle - due to the inclusion of magneto-optical effects. The fictitious iron line at $\lambda 4500$ has $114 \mathrm{~m} \AA$ equivalent width; from left to right and from top to bottom we have displayed patterns Nos. 0, 5, 7, 8, 13, and 19. Angles and field strengths are as in Fig. 3.

does not depend at all on the number of components: there is little difference between the simple triplet and pattern Nos. 19 with 45 components. Pattern No. 9 on the other hand exhibits almost total insensitivity. Such behaviour comes as no surprise. It is clear that the relative strengths and the respective splittings of the Zeeman components determine the magneto-optical intensification, and only sufficient overlap between the $\pi$ - and the $\sigma$-components can create favourable conditions. Table 3 gives details on the magneto-optical intensification of all 20 patterns.

\subsection{Where in the line are magneto-optical effects important?}

We have calculated high resolution Stokes I profiles with and without the magneto-optical terms in the polarised radiative transfer equation. Taking the respective differences between these (field angle and field strength dependent) profiles, one can visualize in which part of the profile and by what amount magneto-optical effects lead to a drop in intensity (decrease in Stokes $I$ ) and a corresponding increase in equivalent width. Figure 6 (bottom panel) displays in detail the highly complex structure of the Stokes I profiles over a $0.6 \AA$ interval as a function of field strength (up to $6 \mathrm{~T}$ ) and for a field angle of $45^{\circ}$, 
Table 2. Characteristics of magnetic intensification of a fictitious $\mathrm{Fe}_{\text {II }}$ line at $4500 \AA$ in a $10000 \mathrm{~K} \log g=4.0$ Kurucz model atmosphere. Column 1 lists the pattern number, $W / W_{0}$ gives the maximum increase over zero field equivalent width, $B_{\max }$ and $\gamma_{\max }$ the field strength (in Teslas) and field angle (in degrees) at which maximum intensification occurs. Results are given for 4 different zero field line strengths with computations not going beyond $13 \mathrm{~T}$.

\begin{tabular}{rrrrrrrrrrrrr}
\hline \hline & & $60 \mathrm{~m} \AA$ & \multicolumn{1}{c}{$83 \mathrm{~m} \AA$} & \multicolumn{3}{c}{$114 \mathrm{~m} \AA$} & \multicolumn{3}{c}{$182 \mathrm{~m} \AA$} \\
Nos. & $W / W_{0}$ & $B_{\max }$ & $\gamma_{\max }$ & $W / W_{0}$ & $B_{\max }$ & $\gamma_{\max }$ & $W / W_{0}$ & $B_{\max }$ & $\gamma_{\max }$ & $W / W_{0}$ & $B_{\max }$ & $\gamma_{\max }$ \\
\hline 0 & 1.4 & 0.8 & 50 & 1.5 & 1.0 & 40 & 1.6 & 1.4 & 42 & 1.5 & 1.6 & 56 \\
1 & 2.1 & 1.6 & 54 & 2.6 & 1.6 & 42 & 2.8 & 2.0 & 46 & 2.5 & 2.4 & 56 \\
2 & 2.9 & $\geq 13.0$ & 60 & 3.7 & $\geq 13.0$ & 52 & 3.6 & $\geq 13.0$ & 26 & 3.0 & $\geq 13.0$ & 22 \\
3 & 2.1 & $\geq 13.0$ & 56 & 2.4 & 12.8 & 52 & 2.4 & 10.2 & 26 & 2.2 & $\geq 13.0$ & 24 \\
4 & 2.6 & $\geq 13.0$ & 58 & 3.0 & $\geq 13.0$ & 50 & 3.0 & $\geq 13.0$ & 24 & 2.5 & $\geq 13.0$ & 22 \\
5 & 3.4 & 8.6 & 62 & 5.1 & 4.4 & 52 & 5.9 & 6.0 & 40 & 5.3 & 8.6 & 38 \\
6 & 2.9 & 5.4 & 60 & 4.0 & 4.2 & 48 & 4.5 & 5.6 & 42 & 4.0 & 6.4 & 50 \\
7 & 3.7 & $\geq 13.0$ & 48 & 5.9 & 7.4 & 56 & 7.0 & 7.4 & 58 & 6.6 & 9.4 & 58 \\
8 & 2.6 & 4.2 & 54 & 3.5 & 3.0 & 60 & 4.1 & 3.8 & 60 & 3.7 & 5.0 & 58 \\
9 & 2.0 & 6.4 & 54 & 2.4 & 9.8 & 54 & 2.4 & 10.6 & 54 & 2.1 & 12.0 & 54 \\
10 & 2.5 & 4.0 & 58 & 3.3 & 3.4 & 42 & 3.7 & 4.0 & 38 & 3.2 & 5.0 & 48 \\
11 & 2.1 & 2.2 & 56 & 2.5 & 1.6 & 60 & 2.7 & 2.0 & 60 & 2.5 & 2.6 & 58 \\
12 & 1.4 & 1.8 & 50 & 1.6 & 2.2 & 38 & 1.6 & 2.6 & 44 & 1.5 & 3.2 & 56 \\
13 & 2.9 & 11.4 & 70 & 4.0 & $\geq 13.0$ & 90 & 4.2 & 2.8 & 64 & 3.6 & 4.0 & 64 \\
14 & 3.1 & 12.4 & 90 & 4.4 & 2.6 & 60 & 4.9 & 2.8 & 56 & 4.3 & 3.6 & 60 \\
15 & 3.3 & $\geq 13.0$ & 50 & 4.7 & 3.8 & 58 & 5.6 & 4.0 & 58 & 5.1 & 5.2 & 58 \\
16 & 3.5 & $\geq 13.0$ & 48 & 5.2 & $\geq 13.0$ & 54 & 5.4 & $\geq 13.0$ & 52 & 4.4 & $\geq 13.0$ & 28 \\
17 & 4.1 & $\geq 13.0$ & 64 & 7.2 & 10.8 & 58 & 9.3 & 10.0 & 42 & 8.8 & 12.0 & 44 \\
18 & 3.7 & $\geq 13.0$ & 62 & 5.4 & $\geq 13.0$ & 56 & 5.4 & $\geq 13.0$ & 34 & 4.6 & $\geq 13.0$ & 22 \\
19 & 4.4 & $\geq 13.0$ & 66 & 8.4 & $\geq 13.0$ & 62 & 10.9 & 9.6 & 50 & 10.3 & 10.8 & 46 \\
\hline
\end{tabular}

Table 3. Similar to Table 2 but with $R=100(W-W n) / W_{n}$ the maximum percental increase in magnetic intensification due to the inclusion of magneto-optical effects.

\begin{tabular}{rrrrrrrrrrrrr}
\hline \hline & \multicolumn{1}{c}{$60 \mathrm{~m} \AA$} & \multicolumn{3}{c}{$83 \mathrm{~m} \AA$} & \multicolumn{3}{c}{$114 \mathrm{~m} \AA$} & \multicolumn{3}{c}{$182 \mathrm{~m} \AA$} \\
Nos. & $R$ & $B_{\max }$ & $\gamma_{\max }$ & $R$ & $B_{\max }$ & $\gamma_{\max }$ & $R$ & $B_{\max }$ & $\gamma_{\max }$ & $R$ & $B_{\max }$ & $\gamma_{\max }$ \\
\hline 0 & 5.6 & 0.6 & 42 & 15.2 & 1.0 & 34 & 19.6 & 1.2 & 42 & 19.4 & 1.4 & 54 \\
1 & 4.3 & 0.6 & 44 & 15.7 & 1.2 & 40 & 24.5 & 1.4 & 52 & 31.0 & 1.8 & 58 \\
2 & 5.7 & 0.6 & 42 & 15.1 & 1.0 & 32 & 20.0 & 1.2 & 42 & 21.0 & 13.0 & 18 \\
3 & 5.6 & 0.8 & 40 & 15.2 & 1.2 & 34 & 20.4 & 1.6 & 40 & 21.7 & 2.0 & 54 \\
4 & 5.5 & 0.8 & 40 & 15.1 & 1.0 & 34 & 19.9 & 1.4 & 40 & 20.2 & 1.6 & 54 \\
5 & 0.8 & 1.0 & 50 & 5.0 & 2.2 & 46 & 10.9 & 5.6 & 32 & 16.0 & 2.2 & 64 \\
6 & 1.0 & 2.2 & 50 & 6.9 & 3.6 & 40 & 14.0 & 5.2 & 32 & 17.8 & 6.0 & 46 \\
7 & 0.4 & 0.8 & 54 & 3.0 & 4.8 & 52 & 11.8 & 6.6 & 54 & 23.2 & 8.6 & 56 \\
8 & 0.9 & 1.8 & 52 & 6.6 & 2.6 & 54 & 18.6 & 3.6 & 56 & 27.7 & 4.4 & 54 \\
9 & 0.2 & 0.4 & 62 & 0.2 & 0.6 & 64 & 0.2 & 0.8 & 62 & 0.2 & 1.2 & 58 \\
10 & 4.5 & 0.8 & 44 & 15.7 & 1.4 & 38 & 25.6 & 2.0 & 48 & 34.5 & 2.4 & 58 \\
11 & 1.8 & 1.0 & 54 & 7.4 & 1.4 & 56 & 15.4 & 1.8 & 58 & 22.6 & 2.4 & 54 \\
12 & 5.7 & 1.4 & 40 & 15.2 & 2.0 & 34 & 19.6 & 2.4 & 42 & 19.4 & 2.8 & 54 \\
13 & 0.4 & 0.6 & 52 & 2.2 & 1.2 & 58 & 6.9 & 1.4 & 62 & 12.0 & 1.8 & 60 \\
14 & 0.8 & 1.0 & 50 & 4.0 & 1.6 & 50 & 8.4 & 1.8 & 58 & 14.9 & 2.0 & 64 \\
15 & 1.7 & 0.6 & 50 & 5.9 & 1.2 & 54 & 16.4 & 2.0 & 58 & 28.0 & 2.6 & 60 \\
16 & 5.3 & 0.4 & 40 & 15.7 & 0.6 & 32 & 21.8 & 0.8 & 36 & 22.9 & 3.4 & 24 \\
17 & 4.0 & 0.8 & 44 & 13.6 & 1.4 & 40 & 24.0 & 3.2 & 44 & 36.0 & 4.6 & 58 \\
18 & 5.6 & 0.4 & 40 & 15.1 & 0.6 & 32 & 19.9 & 0.8 & 38 & 21.2 & 7.6 & 18 \\
19 & 4.1 & 0.4 & 44 & 11.5 & 0.8 & 38 & 17.5 & 2.4 & 38 & 22.2 & 3.4 & 54 \\
\hline
\end{tabular}

whereas the surface shown in the top panel represents the deepening of the profiles due to magneto-optical effects.

The interpretation of Fig. 6 is not a straightforward matter; not all of the structure can be uniquely related to the various Zeeman components and to the regions where they overlap. This is illustrated by cuts through the 2 surfaces, made at $3 \mathrm{~T}$ and at $6 \mathrm{~T}$ as shown in Fig. 7. In the inner part of the intensity profile at $3 \mathrm{~T}$ no correlation can be detected between the various local minima and the positions of the respective Zeeman components. Only the outermost $3 \sigma$-components can 

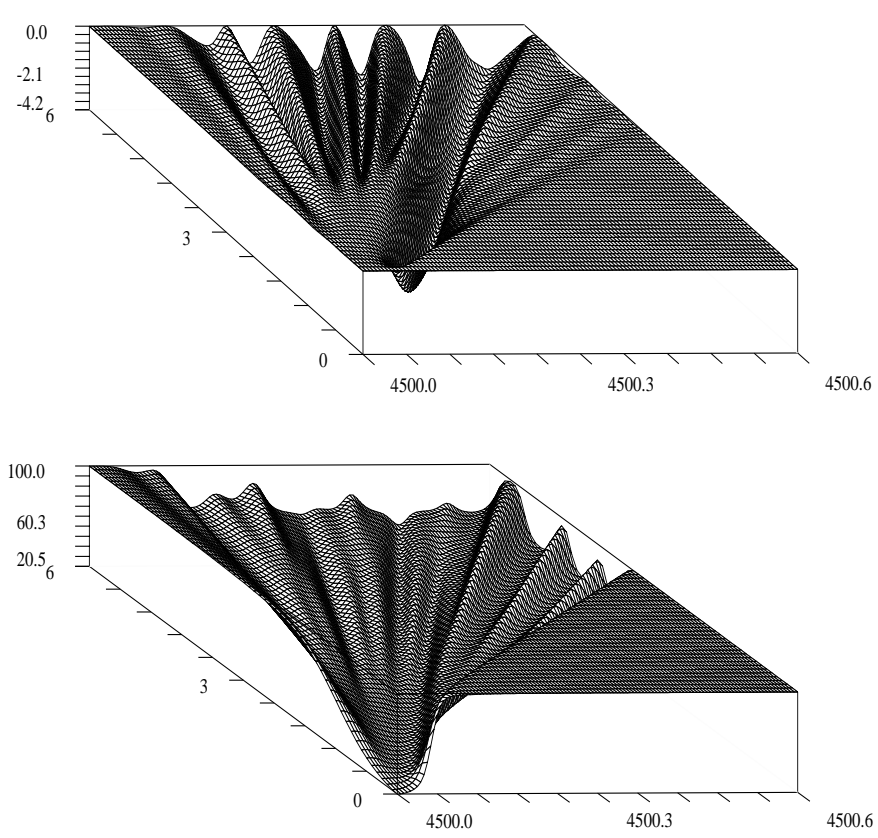

Fig. 6. Bottom: stokes $I$ profiles as a function of magnetic field strength for the $83 \mathrm{~m} \AA$ iron line with Zeeman pattern No. 7. Top: similar, but displaying the difference $\Delta I$ between Stokes $I$ profiles calculated with and without magneto-optical terms. The field angle is $45^{\circ}$, wavelengths are in $\AA$, field strengths in Tesla, $I$ and $\Delta I$ are in \% relative to the continuum.

clearly be identified. In a $6 \mathrm{~T}$ field, with less overlap between the $\sigma$-components and $\pi$-components than in the previous case, the situation improves somewhat, but we note that the deepest minimum occurs right between the strongest $\pi$-components and the second strongest $\sigma$-component.

As to the intensification due to magneto-optical effects, the structure is less complicated and for the (weak) inner Zeeman components we see a very good correlation between the positions of the Zeeman components and the drop in intensity (Stokes $I$ ). At $6 \mathrm{~T}$, intensification increases with the strength of the Zeeman components, reaching maximum importance where the strongest $\pi$-component overlaps with the strongest $\pi$-component. The latter is also true for a $3 \mathrm{~T}$ field.

We conclude that intensification due to magneto-optical effects is difficult if not impossible to predict, since it depends, in a very sensitive way, on the Zeeman splitting and on the relative intensities of the $\pi$ - and the $\sigma$-components. Very good wavelength resolution is needed to resolve the fine structure of magnetically broadened and intensified lines.

\section{Magnetic intensification and line blanketing}

As we have seen, magnetic intensification can lead to large changes in equivalent width of lines with complex Zeeman patterns; it can manifest itself quite dramatically in narrow wavelength intervals and in the case of exotic Zeeman patterns. But how does blending in crowded spectra affect intensification, what does the surface intensification vs. field strength and angle look like? Will there be observable photometric variations in Johnson broad-band photometry with intervals of typically $1000 \AA$ ? We cannot advance more than the educated guess that

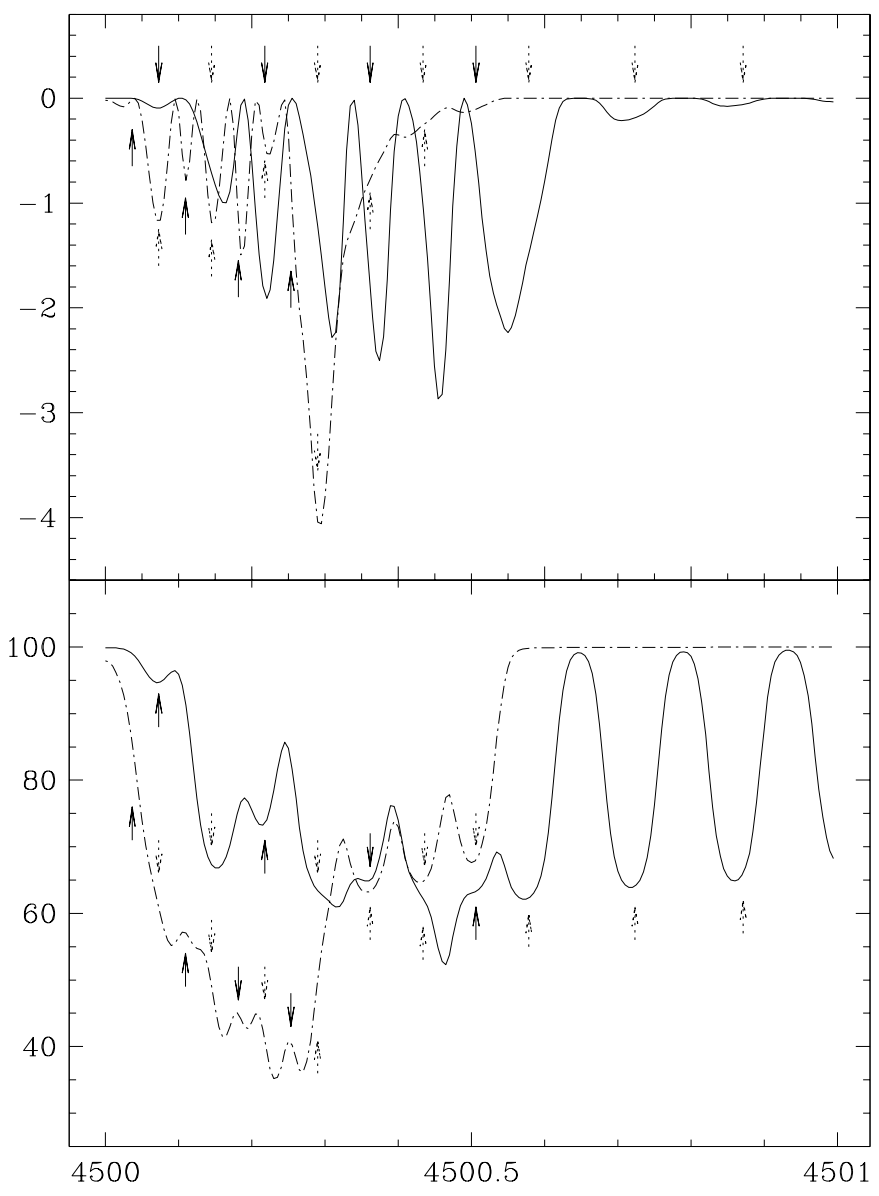

Fig. 7. Bottom: cut through the Stokes $I$ surface in the bottom of Fig. 6 at $3 \mathrm{~T}$ (dash-dotted line) and at $6 \mathrm{~T}$ (full line). The respective positions of the Zeeman components are indicated by full $(\pi)$ and dash-dotted $(\sigma)$ arrows. Top: similar, but representing a cut through the intensification surface in the top of Fig. 6. The arrows in the uppermost part pertain to the $6 \mathrm{~T}$ field, the rest to the $3 \mathrm{~T}$ field.

as long as line blocking does not become excessive, intensity (Stokes I) will probably decrease only moderately; blending could have a smoothing effect.

We have extended our investigation of single iron lines with various Zeeman patterns discussed in the previous sections to a full spectrum over $500-1000 \AA$ intervals in a $10000 \mathrm{~K}$, $\log g=4.0$ Kurucz model atmosphere, using normal solar abundances, but also abundances of $\mathrm{Sc}, \mathrm{Ti}, \mathrm{V}, \mathrm{Cr}, \mathrm{Mn}, \mathrm{Fe}, \mathrm{Co}$, and $\mathrm{Ni}$ enhanced by $1 \mathrm{dex}$. For reasons of CPU time, we cannot afford to calculate the same dense field strength and angle grid as in the study of single lines, but as it turns out there are no details in the Stokes $I$ surfaces that would require such high resolution.

Figure 8 displays the results for the interval 3800-4300 , both for normal (top) and enhanced abundances (bottom). The respective surfaces are extremely smooth, the maximum decrease in integrated intensity (Stokes $I$ ) goes from $-0.8 \%$ for normal abundances to $-3.6 \%$ for enhanced metallicity. Corresponding line blocking is a modest $1.7 \%$ in the former, $4.7 \%$ in the latter case (both without hydrogen lines), $17.1 \%$ and $19.5 \%$ respectively with hydrogen lines; maximum magnetic intensification (increase in total equivalent width) is $4.0 \%$ 

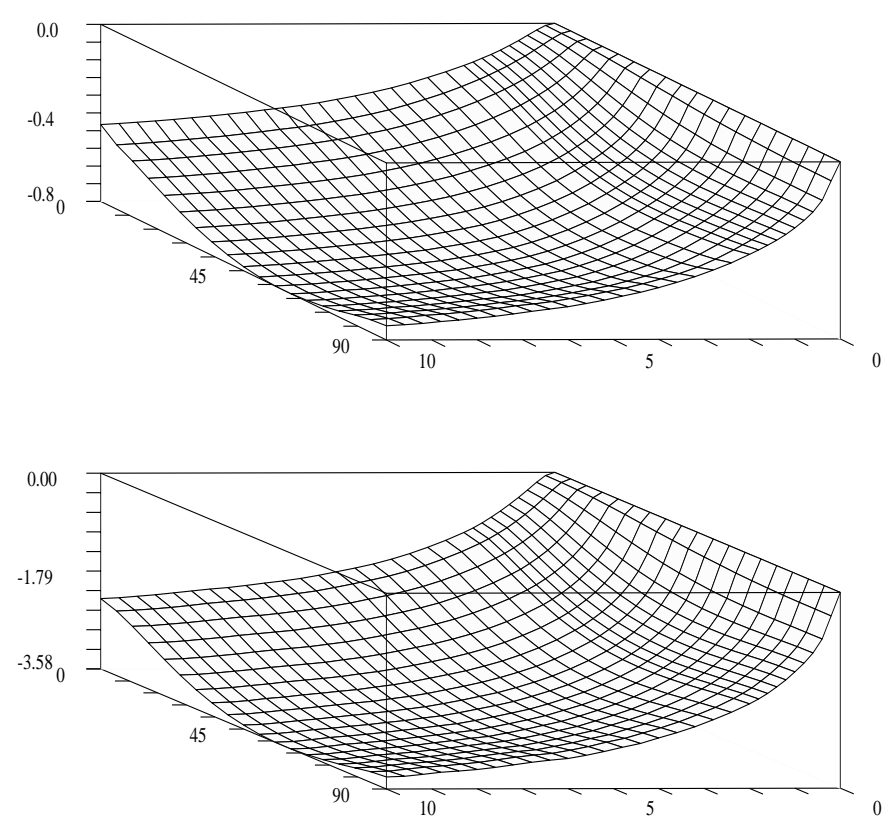

Fig. 8. Top: percental decrease in intensity (Stokes $I$ ) integrated over the spectral interval $3800-4300 \AA$ as a function of magnetic field strength and field angle for solar chemical abundances. Bottom: the same but for the abundances of $\mathrm{Sc}, \mathrm{Ti}, \mathrm{V}, \mathrm{Cr}, \mathrm{Mn}, \mathrm{Fe}, \mathrm{Co}$, and $\mathrm{Ni}$ enhanced by 1 dex.

and $15.6 \%$. For all practical purposes, the very small influence of magneto-optical effects - of the order of $0.1 \%$ - thus becomes negligible.

Similar results were obtained for other wavelength intervals. In the case of solar abundances there is a consistent $0.7-$ $0.8 \%$ maximum decrease in intensity (Stokes $I$ ) for 3 intervals investigated, viz. 3500-4500, 4250-4750 and 4050-4950 A. These already small values drop by about $2 / 3$ to $0.25-0.30$ if we take all lines to be simple Zeeman triplets.

We conclude that in CP star atmospheres with large metal over-abundances, magnetic intensification leads to a moderate decrease in the intensity spectrum (Stokes $I$ ) in the blue and in the visible. Assuming a magnetic field configuration characterised by a dipole inside the star whose axis is not aligned with the rotation axis, we expect the integrated intensity signal to vary with the rotational phase. However, as Fig. 8 suggests, the amplitude of these variations will be rather small. Taking the Stokes $I$ surface for enhanced metal abundances displayed in the bottom of Fig. 8 and carrying out a Monte Carlo search in the parameter space of Stift's (1975) oblique rotator model, we never have detected amplitudes in excess of $1 \%$. So magnetic intensification could conceivably be responsible for at least part of the observed small-amplitude light variations of magnetic stars, but one has to keep in mind that any modification of the atmospheric structure due to magnetic forces and/or diffusion driven stratification of chemical elements could be of the same order of magnitude. See also Leone \& Catanzaro (2001) for a discussion for the relation between luminosity and magnetic field variations.
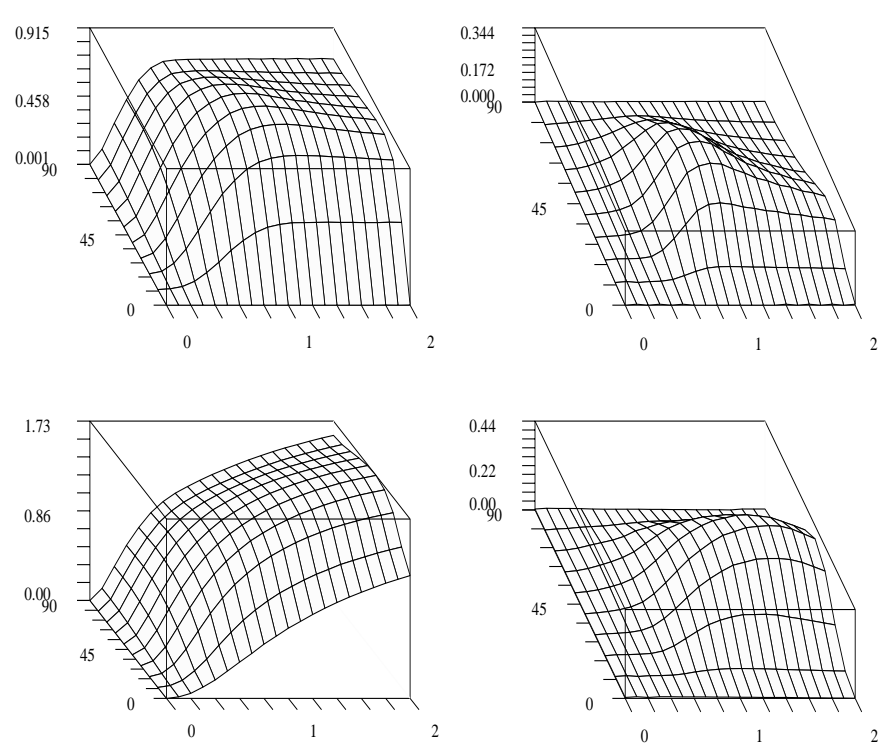

Fig. 9. Left: virtual over-abundances (in dex) as a function of field strength (in Tesla) and field angle as derived from the $83 \mathrm{~m} \AA$ iron line with Zeeman pattern No. 0 (top) and pattern No. 10 (bottom). Right: similar, but displaying abundance errors resulting from modelling the line without anomalous dispersion terms.

\section{Magnetic intensification and abundance determinations}

From our results it emerges that abundance determinations can be severely affected by magnetic intensification. Figure 9 illustrates the magnitude of the virtual over-abundances as a function of field strength and field angle, derived from the $83 \mathrm{m \AA}$ iron line with Zeeman patterns Nos. 0 and 10 respectively. Even for a simple triplet, Zeeman splitting can give rise to an apparent 0.92 dex over-abundance at field strengths of about 1 Tesla. Zeeman pattern No. 10 with 9 components is much more sensitive to intensification, yielding $1.73 \mathrm{dex}$ at 2 Tesla, the maximum being encountered far beyond that value. It has to be noted that for patterns Nos. 7, 8, 13, 14, 15, and 19 the magnetically enhanced equivalent widths at $2 \mathrm{~T}$ (and sometimes even lower) cannot be reproduced under the assumption of zero magnetic field since they do not intersect with the respective curves of growth at any value of over-abundance.

Landolfi et al. (1989) have shown that it is important to include magneto-optical effects in order to correctly interpret Stokes profiles in CP stars, a point also made by Wade et al. (2001). So we had a look at the influence of magneto-optical effects on virtual abundances. Figure 9 reveals that anomalous dispersion accounts for up to 0.34 dex in the triplet case, up to 0.44 dex for pattern No. 10 . We can thus confirm that any modelling of stellar spectra without magneto-optical terms in the radiative transfer equation must lead to an overestimate of elemental abundances even if the magnetic model were correctly known. 


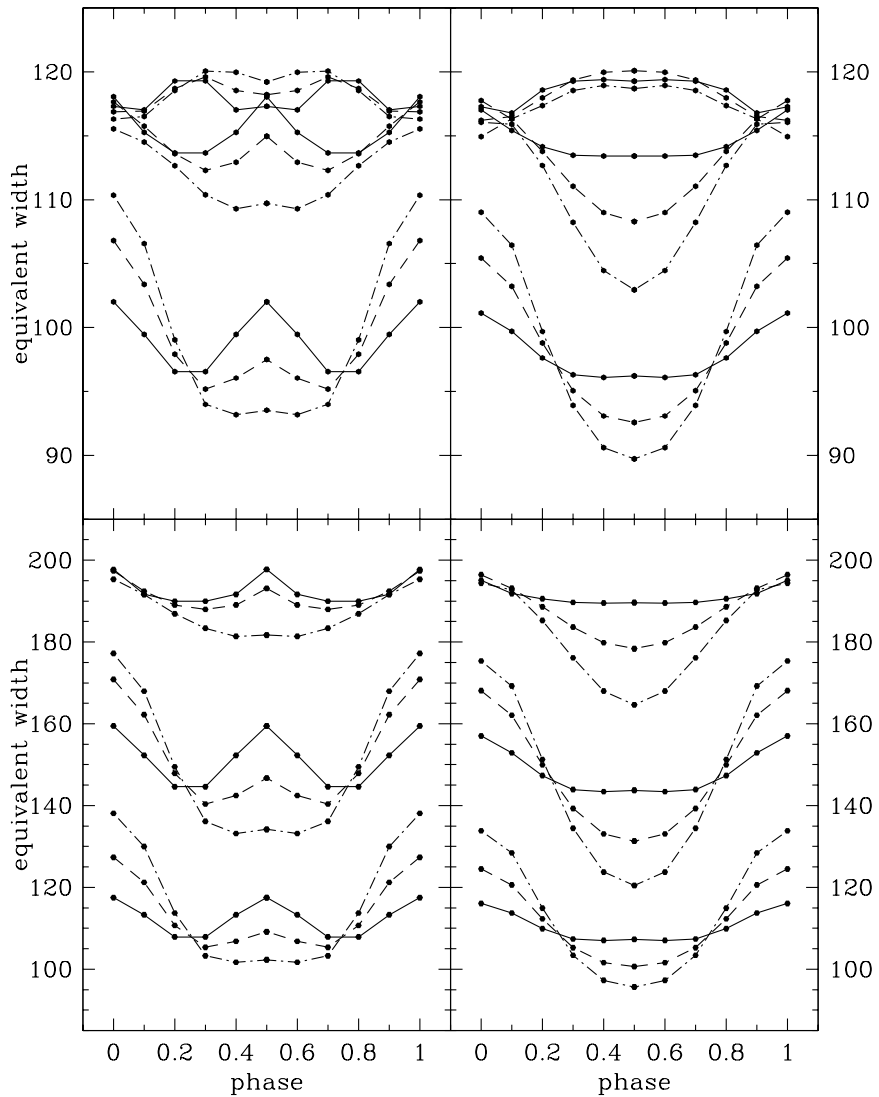

Fig. 10. Equivalence width variations of the $83 \mathrm{~m} \AA$ iron line with Zeeman patterns No. 0 (top panels) and No. 1 (bottom panels) for an Oblique Rotator Model with inclination $i=90^{\circ}$ and obliquity $\alpha=-90^{\circ}$ (left), $i=60^{\circ}$ and $\alpha=-40^{\circ}$ (right). Full lines correspond to a centred dipole $x_{2}=0.0$, dashed lines to $x_{2}=0.1$ and dash-dotted lines to $x_{2}=0.2$ - for the definition of these parameters see Stift (1975). The groups of 3 curves each pertain to $0.25 \mathrm{~T}, 0.50 \mathrm{~T}$, and $1.0 \mathrm{~T}$ dipole momentum (from bottom to top), corresponding to $0.50 \mathrm{~T}$, and $1.0 \mathrm{~T}$ and $2.0 \mathrm{~T}$ polar field strength of the centred dipole.

\subsection{Virtual over-abundances and virtual abundance variations in magnetic stars}

In magnetic stars with dipolar or complex dipolar-quadrupolar surface magnetic field configurations the resulting magnetic intensification integrated over the visible hemisphere will vary with rotational phase. We therefore expect to observe equivalent width variations which depend on Zeeman pattern, magnetic field geometry and magnetic field strength. It is impossible to make general predictions, but modelling of simple Oblique Rotator configurations can give some hints as to the occurrence and the amplitude of such variations.

Figure 10 shows that, in accord with what one can infer from Fig. 3, substantial, readily observable variations in equivalent width occur already at moderate field strengths. As an illustration, for the model with inclination $i=90^{\circ}$, obliquity $\alpha=-90^{\circ}$ and decentring parameter $x_{2}=0.2$ (see Stift 1975 for the definition of the parameters), equivalent widths for Zeeman pattern No. 1 vary between about $100-140 \mathrm{~m} \AA$, the longitudinal field from -0.140 to $+0.165 \mathrm{~T}$ and the field modulus from 0.28 and $0.59 \mathrm{~T}$. These variations first increase with

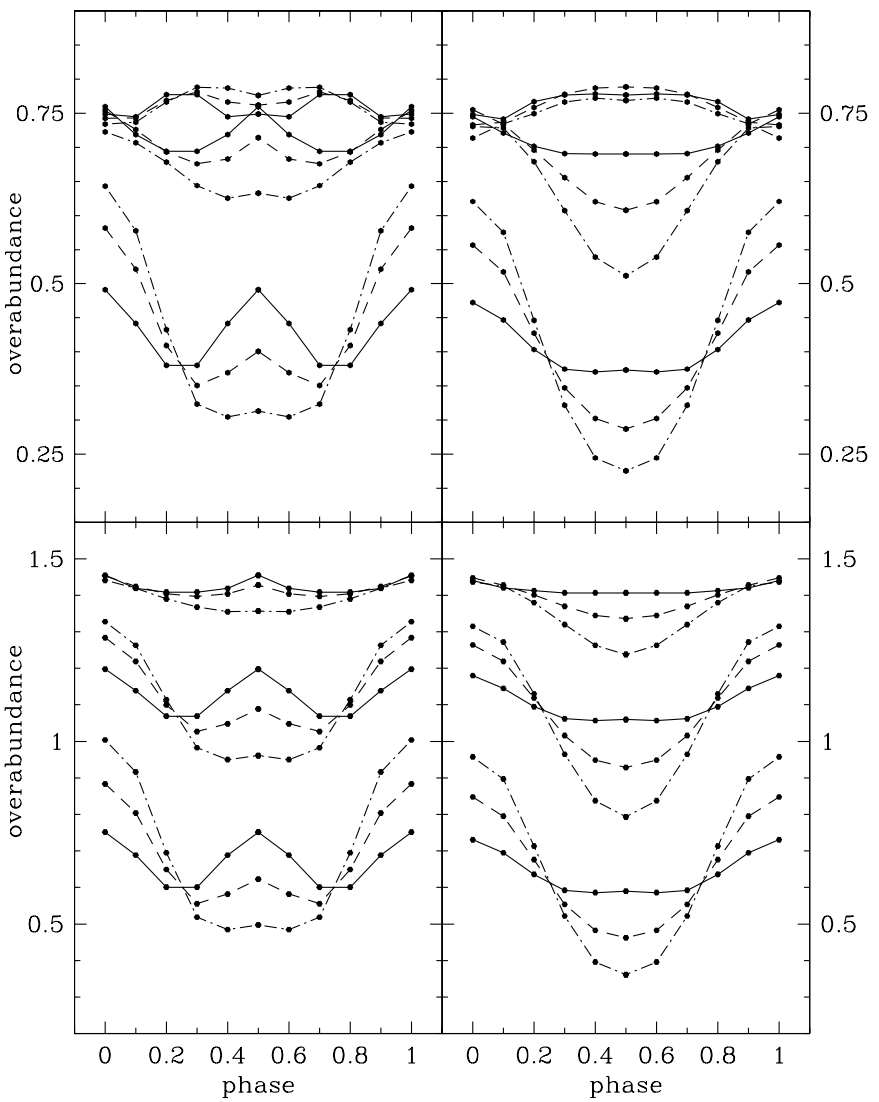

Fig. 11. Similar to Fig. 10 but displaying the corresponding virtual over-abundances.

field strength but tend to decrease again once magnetic desaturation remains more or less constant. The corresponding virtual over-abundances are displayed in Fig. 11.

It is interesting to verify whether the predictions of our calculations in conjunction with the magnetic models of 4 Ap stars published by Stift \& Goossens (1991) are in accord with observational evidence. In HD 215441, Babcock (1960) noticed a "subtle type of spectrum variability", in HD $137909(\beta \mathrm{CrB})$ Mathys (1991) found no significant equivalence width variations in most spectral lines apart from the $\mathrm{Cr}$ II lines which appear to be marginally variable. Preston (1970), in his study of HD 126515, derived pseudo-equivalent width variations - defined as the product of the central depth of a line and the width at half depth - for several elements which typically vary by a factor of 2. Mathys (1991) confirms such variations but does not give any details. Finally, photographic spectra of HD 65339 (53 Cam) have been analysed by Faraggiana (1973) who gives plots of mean intensity variations of selected multiplets of singly ionised $\mathrm{Ti}, \mathrm{Cr}$, and $\mathrm{Fe}$. We conjecture that these mean intensities are somehow related to the pseudo-equivalent widths of Preston as defined above.

As it turns out, the magnetic models of Stift \& Goossens (1991) yield, for most Zeeman patterns, equivalent width variations of the order of only about 5\%. Pattern No. 1 behaves in an exceptional way with an amplitude that can reach $20 \%$ as in the case of the low-excentricity model for HD 126515 (see Fig. 12). Although this is far from the factor of 2 found 


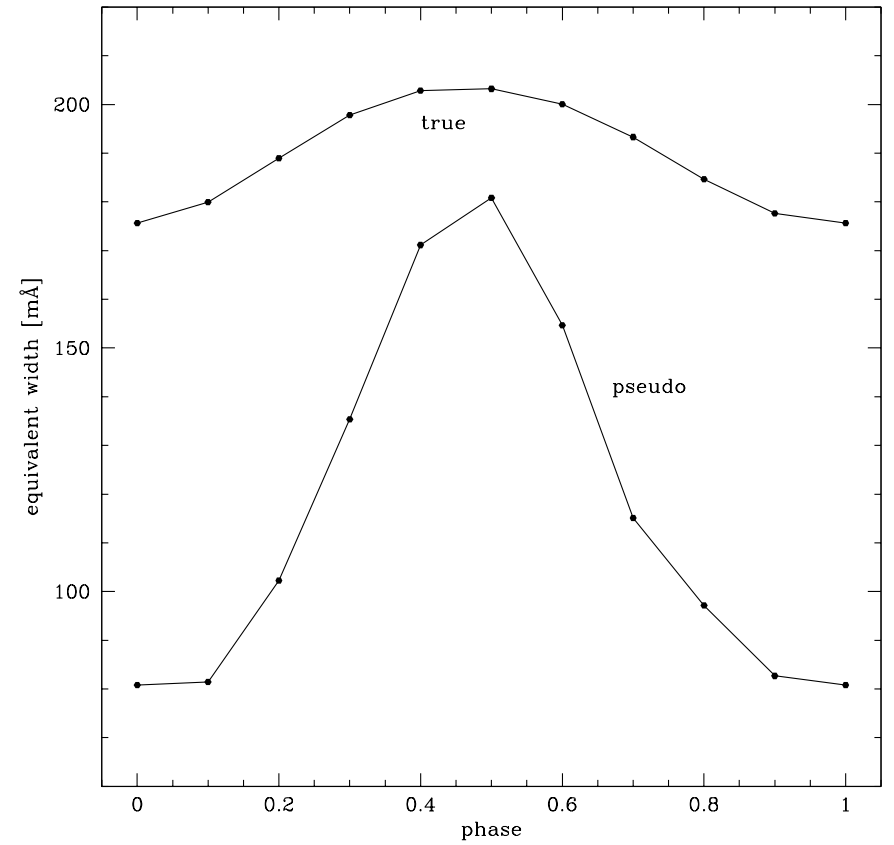

Fig. 12. True and pseudo-equivalence width variations predicted for Zeeman pattern No. 1, based on the low-excentricity model of HD 126515 in Stift \& Goossens (1991).

by Preston's (1970), there is not contradiction with his results since the pseudo-equivalent width exhibits the desired much larger variations.

Figure 13 displays - for 4 different Zeeman patterns - the variations in virtual over-abundances as they would result from the models of Stift \& Goossens (1991). Whereas abundance variations always remain below $0.2 \mathrm{dex}$, abundances themselves can be overestimated (depending on the lines chosen) by between 0.5 and $1.2 \mathrm{dex}$ as in the case of $\beta \mathrm{CrB}$ or 0.7 to 1.45 dex as in the case of Babcock's star.

There are several ways to account in a rough way for the effects of magnetic intensification on abundance determinations in CP stars. Such methods are solely justified in those cases where longitudinal field observations only are available. One possible approach consists in looking for the best centred dipole fit to the longitudinal field variations and to take into account the corresponding magnetic geometry in the subsequent abundance analysis. However, the models derived this way are non-unique and the probability is high that the results of the analysis will still be substantially in error. Taking the example of $\beta \mathrm{CrB}$, the longitudinal field variations predicted by the models of Stift \& Goossens (1991) can well be represented by a centred dipole model with inclination $i=45^{\circ}$, obliquity $\alpha=70^{\circ}$ and polar field strength $0.4 \mathrm{~T}$. Instead of the virtual over-abundances displayed in Fig. 13, viz. 0.55-0.62 dex for pattern No. 0 and $0.87-0.97$ dex for pattern No. 1 our centred dipole model now predicts $0.28-0.30$ dex and $0.45-0.47$ dex respectively. The error is halved but still substantial.

The only other scheme worth mentioning consists in mimicking the effects of Zeeman splitting by an enhanced microturbulence (Hensberge 1974) but an in-depth assessment of the reliability of this method is far outside the scope of this paper.

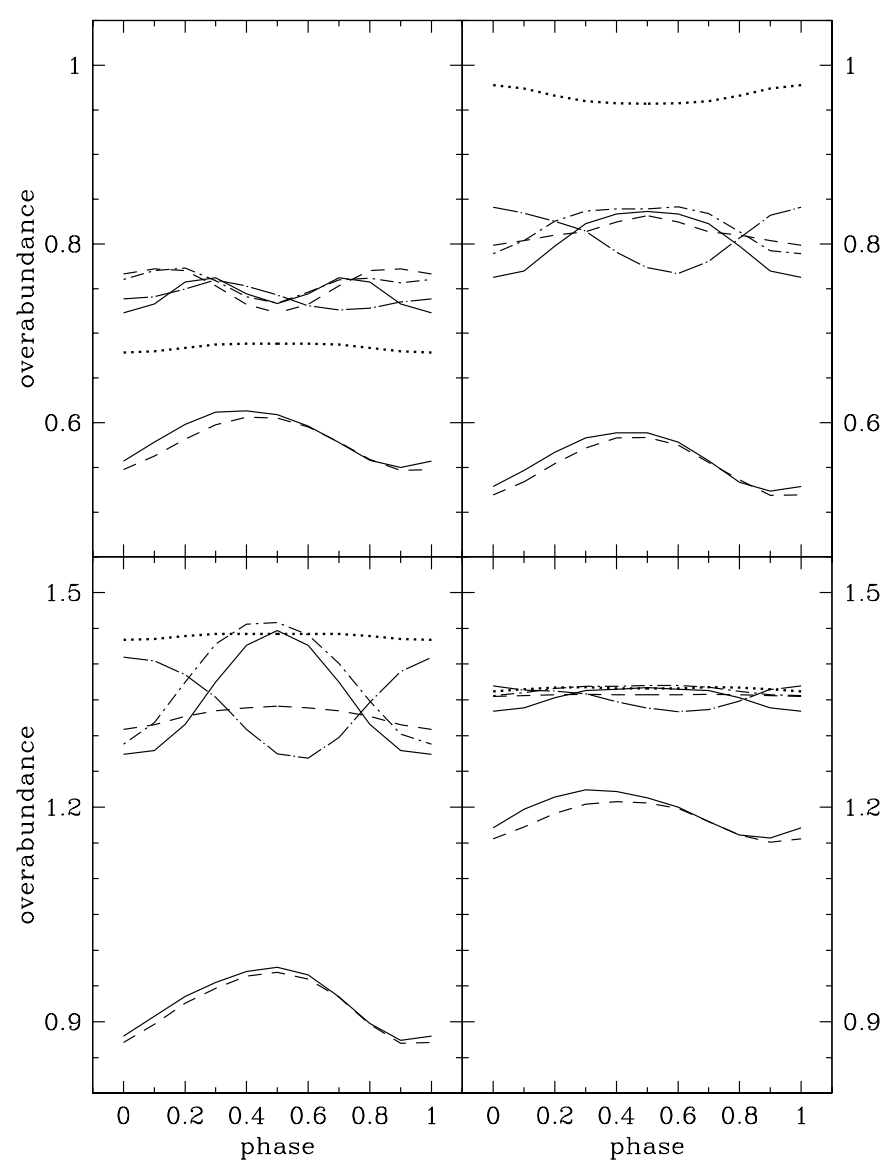

Fig. 13. Virtual over-abundances derived from the $83 \mathrm{~m} \AA$ iron line with respective Zeeman patterns Nos. 0, 4, 1, 9 (from left to right and from top to bottom). The magnetic models have been taken from Stift \& Goossens (1991). The lowest curves pertain to model 1 (solid) and model 2 (dashed) of HD $137909(\beta \mathrm{CrB})$, the dotted curve to HD 215441 (Babcock's star). In addition there are model 1 (solid) and model 2 (dashed) of HD 65339 (53 Cam) and model 1 (dot - short dash) and model 2 (dot - long dash) of HD 126515. Note that in Stift's model the curves can be arbitrarily shifted in phase.

\section{Conclusions}

We have carried out an extensive investigation of the effects of Zeeman splitting and of polarised radiative transfer on the equivalent widths of spectral lines, the so called "magnetic intensification". Our results show that the increase in equivalent width can be fairly large, reaching values in excess of 10, but that in complex Zeeman patterns it always falls well below the $n / 2$ relation (where $n$ is the number of Zeeman components) suggested by Babcock (1949). We also confirm that magnetooptical effects can be quite important at certain field angles and field strengths. Line-blanketing is also affected to some degree, but the decrease in intensity (Stokes $I$ ) is rather small and the ensuing light variations can possibly be masked by other effects like the modification of the atmospheric structure by magnetic forces and/or by radiation driven diffusion and ensuing stratification of chemical elements.

Finally, we have addressed the question of how magnetic intensification affects abundance determination in magnetic 
stars. For simple oblique rotator models with dipolar field configurations we predict easily detectable equivalence width variations for a number of Zeeman patterns; spurious overabundances can reach values from $0.5-1.5 \mathrm{dex}$ in the 4 wellknown magnetic stars modelled by Stift \& Goossens (1991). This suggests that any investigation of the relation between the magnetic field structure of CP stars with fairly strong magnetic fields and their relation to element surface distributions derived without due regard to Zeeman intensification can hardly be expected to yield reliable results. The same argument holds for simple Doppler Imaging of these stars based solely on intensity spectra.

Acknowledgements. MJS acknowledges support by the Austrian Science Fund $(F W F)$, project P12101-AST "Solar and Stellar Magnetic Polarisation". Thanks go to Egidio Landi Degl'Innocenti for numerous helpful discussions (or rather lectures) on the workings of polarisation.

\section{References}

Alecian, G., \& Stift, M. J. 2002, A\&A, 387, 271

Auer, L. H., Heasley, J. N., \& House, L. L. 1977, ApJ, 216, 531

Babcock, H. W. 1949, ApJ, 110, 126

Babcock, H. W. 1960, ApJ, 132, 521

Faraggiana, R. 1973, A\&A, 22, 265

Hensberge, H., \& Loore, C. 1974, A\&A, 37, 367
Kurucz, R. 1993, CDROM Model Distribution, Smithsonian Astrophys. Obs.

Landi Degl'Innocenti, E. 1987, in Numerical Radiative Transfer, ed. W. Kalkofen (Cambridge Univ. Press), 265

Landolfi, M., Landi degl'Innocenti, M., \& Landi degl'Innocenti, E. 1989, A\&A, 216, 113

Landolfi, M., Landi Degl'Innocenti, E., Landi Degl'Innocenti, M., \& Leroy, J.-L. 1993, A\&A, 272, 285

Leone, F., \& Catanzaro, G. 2001, A\&A, 365, 118

Leroy, J. L. 1962, Ann. Ap., 25, 127

Leroy, J. L. 1989, A\&A, 215, 360

Leroy, J. L. 1995, A\&AS, 114, 79

Leroy, J. L., Landolfi, M., \& Landi degl'Innocenti, E. 1993, A\&A, 270,335

Mathys, G. 1991, A\&AS, 89, 121

Preston, G. W. 1970, ApJ, 160, 1059

Rees, D. E. 1987, in Numerical Radiative Transfer, ed. W. Kalkofen (Cambridge Univ. Press, Cambridge), 213

Stift, M. J. 1975, MNRAS, 172, 133

Stift, M. J., \& Goossens, M. 1991, A\&A, 251, 139

Stift, M. J. 1996, in Stellar Surface Structure, ed. K. G. Strassmeier, \& J. L. Linsky (Kluwer, Dordrecht), IAU Symp., 176, 61

Stift, M. J. 1998a, Comp. Phys., 12, 150

Stift, M. J. 1998b, in Reliable Software Technologies - Ada Europe '98, ed. L. Asplund, Lect. Notes Comp. Sci., 1411, 128

Stift, M. J. 2000, A Pecul. Newslett., 33

Wade, G. A., Bagnulo, S., Kochukhov, O. P., et al. 2001, A\&A, 374, 265

Warwick, J. W. 1951, AJ, 56, 51 